\title{
The time course of orthography and phonology: ERP correlates of masked priming effects in Spanish
}

\author{
MANUEL CARREIRAS,,${ }^{1,2}$ MANUEL PEREA, ${ }^{3}$ MARTA VERGARA, ${ }^{2}$ AND \\ ALEXANDER POLLATSEK ${ }^{4}$ \\ ${ }^{1}$ Basque Center on Cognition, Brain and Language, Donostia, Spain \\ ${ }^{2}$ Instituto de Tecnologías Biomédicas, Universidad de La Laguna, La Laguna, Spain \\ ${ }^{3}$ Departamento de Metodología, Universitat de València, València, Spain \\ ${ }^{4}$ Department of Psychology, University of Massachusetts at Amherst, Amherst, Massachusetts, USA
}

\begin{abstract}
One key issue for computational models of visual-word recognition is the time course of orthographic and phonological information during reading. Previous research, using both behavioral and event related brain potential (ERP) measures, has shown that orthographic codes are activated very early but that phonological activation starts to occur immediately afterward. Here we report an ERP masked priming experiment in Spanish that investigates this issue further by using very strict control conditions. The critical phonological comparison was between two pairs of primes having the same orthographic similarity to the target words but differing in phonological similarity (e.g., conal$C A N A L$ vs. cinal-CANAL vis à vis ponel-PANEL vs. pinel-PANEL), whereas the critical orthographic contrast was between pairs of primes that had the same phonological similarity to the target but differing in orthographic similarity (e.g., conal-CANAL vs. konal-CANAL). Orthographic priming was mainly observed in the 150-250-ms time window whereas phonological priming occurred in the $350-550-\mathrm{ms}$ window.
\end{abstract}

Descriptors: Visual word recognition, ERP, Orthography and phonology

One critical issue for any model of visual word recognition and reading is the specification of the role of phonology during lexical access. To examine the influence of phonological effects during lexical access, many researchers have focused on priming procedures. Furthermore, to minimize potential postaccess phenomena, researchers have often opted to mask the prime in order to make it largely unavailable for conscious report. Using such a masked priming procedure, a number of studies have shown that a briefly presented (and masked) homophonic prime (e.g., rait) speeds responses times to a target (e.g., RATE) more than an orthographic control (e.g., raut) in laboratory word identification tasks such as lexical decision (see Rastle \& Brysbaert, 2006, for a recent review). We should note here that Ferrand and Grainger (1994) were the first to use phonological controls to measure masked orthographic priming (e.g., the French pseudohomophones mert-MERE and mair-MERE; see also Ziegler, Ferrand, Jacobs, Rey, \& Grainger, 2000, for another time-course analysis with this type of stimuli). The preferred word laboratory task with this procedure is the lexical decision task, rather than

The research reported in this article has been partially supported by grants PSI2008-04069/PSIC, SEJ2006-09238, and CONSOLIDERINGENIO 2010 (CSD2008-00048) from the Spanish Ministry of Science and Innovation.

Address reprint requests to: Manuel Carreiras, Basque Center on Cognition, Brain and Language, Paseo Mikeletegi, 53, 20009 Donostia, Spain. E-mail: m.carreiras@bcbl.eu the naming task, because the naming task may have an intrinsic phonological component independent of lexical access.

Likewise, in the context of normal silent reading, (a) a parafoveal preview of a word that was a homophone of the target word speeded processing of the target word when it was later fixated (i.e., reduced fixation time on the target) more than an orthographic control (Pollatsek, Lesch, Morris, \& Rayner, 1992), and (b) a homophone "fast prime" presented during the first 30-40 ms of a fixation and then replaced by the target word speeded fixation time on the target word relative to an orthographic control (see Lee, Rayner, \& Pollatsek, 1999). In these paradigms, the primes are rarely, if ever, consciously processed. All these procedures, however, rest on the assumption that the homophone and the control are equally orthographically similar to the target. Although the controls are usually matched with the homophones on a number of variables, such as the number of letters they share with the target, it is usually not possible to control every factor, including the visual similarity of the letters to those in the target word.

In a recent study, Pollatsek, Perea, and Carreiras (2005) employed a new paradigm in which the visual and orthographic similarity between priming conditions was perfectly controlled at the letter level. For the experimental pairs of items, Pollatsek and colleagues (2005) selected pairs of experimental primes that had the same degree of letter overlap with the target while differing in their phonological overlap (e.g., conal-CANAL vs. cinal$C A N A L)$. That is, conal is more phonologically similar to 
$C A N A L$ than cinal is, even though they each differ from $C A N A L$ by one vowel letter. Relying in this comparison as an indication of a phonological effect, however, rests on the assumption that $o$ and $i$ are equally orthographically and visually similar to $A$. As a control for the possibility that this assumption is false, Pollatsek et al. (2005) used pairs of control primes (e.g., ponel-PANEL vs. pinel- $P A N E L)$ in which the same vowel letters were changed as in the experimental primes and thus the orthographic differences in the two pairs should be identical. However, the critical experimental pairs differed in both the first and second phoneme of the word whereas the control pairs differed only in the second phoneme. Thus, any difference in the data in the two cases can unambiguously be ascribed to differences in phonology. Pollatsek et al. (2005) reported that lexical decision times to a target word like $C A N A L$ were faster when it was preceded by a one-letter different nonword prime that shared all phonemes but one (con$a l)$ than when it was preceded by a one-letter different nonword prime that shared all phonemes but two (cinal), whereas lexical decision times to a target like PANEL were virtually identical with ponel and pinel as primes. These results strongly suggest that there is a phonological priming effect in masked priming that cannot be due to uncontrolled orthographic differences. Note that Pollatsek et al. (2005) used Spanish stimuli because the manipulation to be described below is clearer in Spanish than in English (e.g., unlike English, in which many nonstressed vowels are pronounced as schwa, there is no vowel reduction in Spanish).

The main goal of the present experiment is to examine the time course of phonological priming effects on lexical access with the paradigm introduced by Pollatsek et al. (2005) by using electrophysiological measures. Bear in mind that this paradigm completely controls for visual and orthographic differences between experimental and control pairs so that any difference in the pattern of priming results for those pairs has to be due to phonology. For comparison purposes, we also examine the time course of orthographic priming. The rationale of using electrophysiological measures is that response times may not be the best method to directly tap into the time course of processing, because they give the researcher only one data point at the end of processing. Event related potentials (ERPs) are voltage changes recorded from the scalp and extracted from the background electroencephalogram by averaging time-locked responses to stimuli onset. ERPs are functionally decomposable to a greater extent than behavioral data, thus enabling us to draw conclusions not only about the existence of phonological processing, but more importantly, about the precise timing of the underlying phonological processing.

Several recent ERP studies have shown that orthographic and phonological differences between masked primes and targets appear to modulate the ERP signal on target words (e.g., Grainger, Kiyonaga, \& Holcomb, 2006; Holcomb \& Grainger, 2006). More specifically, the amplitude of two components has been shown to be sensitive to the relation between prime and target: The N250 and N400 components showed larger negativities for unrelated prime/target pairs than for related prime/target pairs. Grainger, Kiyonaga, et al. (2006) presented targets preceded by transposed-letter primes (e.g., barin-BRAIN) or their controls (e.g., bosin-BRAIN). Words preceded by a replaced letter control prime produced larger amplitudes than the words preceded by a transposed-letter prime in the 150-250-ms window, but not in later windows. Grainger, Kiyonaga, et al., (2006) interpreted this pattern as reflecting orthographic sublexical processes. Grainger, Kiyonaga, et al., (2006) assumed that transposed-letter priming effects reflect only orthographic processing, as suggested by
Perea and Carreiras (2006, 2008); however, the replacement letters in the control primes could also be considered to be more phonologically different from the target letters than those in transposed-letter primes (e.g., $o$ is more different from $A$ than $a$ is). (See Frankish \& Turner, 2007, for a similar view.)

In addition, Grainger, Kiyonaga, et al. (2006) investigated phonological priming comparing pseudohomophone primes with control primes (e.g., brane-BRAIN vs. brant-BRAIN). Pseudohomophones produced smaller amplitudes than control primes only at the $250-350-\mathrm{ms}$ and $350-550$-ms ERP windows. They interpreted this pattern as reflecting phonological sublexical processes. Thus, the data from Grainger, Kiyonaga, and colleagues (2006) reveal that orthographic and phonological effects seem to have a different time course, with orthographic effects arising earlier than phonological effects. In addition, they found a different topographical distribution for the two effects: Whereas the effect of transposed-letter manipulation in the 150250 -ms window was located in posterior regions, the pseudohomophone effect in the 250-350-ms window was located in anterior regions. Furthermore, other studies have found a modulation of early ERP components by sublexical variables in similar time windows (Carreiras, Vergara, \& Barber, 2005; Carreiras, Gillon-Dowens, Vergara, \& Perea, 2009; see Barber \& Kutas, 2007, for a review of ERP effects in visual word recognition). In this light, Holcomb and Grainger (2007; see also Barber \& Kutas, 2007) mapped the ERP time-course data onto the hypothesized cascade of processes described in the bimodal interactive-activation model of word recognition (see Grainger \& Ferrand, 1994): The early components (e.g., N250) would reflect the mapping of prelexical orthographic representations onto whole-word orthographic representations (and also prelexical mapping of orthography onto phonology), whereas the earliest part of the N400 component was thought to reflect the mapping of whole-word representations onto semantics.

One potential limitation of the Grainger, Kiyonaga, et al. (2006) study is that the phonological priming effect was based on the comparison of a pseudohomophone condition (brane$B R A I N$ ) and its control (e.g., brant-BRAIN). As indicated earlier, this manipulation rests on the assumption that the homophone and the control are equally orthographically similar to the target word - an assumption that may be wrong. To examine the effect of phonology in the present article, we compare pairs like conal-CANAL versus cinal-CANAL, so that the two pairs are perfectly matched in orthography. As a further control, we include pairs like ponel-PANEL versus pinel-PANEL, in which the two critical vowels ( $o$ and $i$ ) are the same, but they do not alter the pronunciation of the letter $p$. In addition, to examine the role of orthography, we employ pairs with the same pronunciation, but a different orthography, like konal-CANAL versus conal$C A N A L$. These primes are thus matched on phonological similarity to the target; however, any observed difference between $k$ and $c$ as primes may not be due to their similarity to the target letter $C$ at an abstract letter level, but may instead be due to differing visual similarity to the target letter. Note that, unlike the present manipulation, orthographic priming effects in most previous studies compared an orthographically related condition versus a completely unrelated condition (e.g., lonc- $L O N G$ vs. tabe-LONG; see Ferrand \& Grainger, 1992; but see Ferrand \& Grainger, 1994), so that factors other than orthography may have influenced those findings.

Based on findings reported by Grainger, Kiyonaga, et al. (2006), there should be a dissociation between orthographic and 
phonological priming effects - mainly in terms of their particular time course. On the one hand, we would expect effects of orthography in the ERP waves with larger negativities for the grapheme-change than for the grapheme-same pairs. On the other hand, if there is early access to phonology, we would expect larger negativities for those ERPs that are supposed to reflect phonological processing, for the condition of phoneme-change (cinal-CANAL) compared to both the phoneme and graphemesame (conal-CANAL) in the experimental pairs, but not in the control pairs (i.e., same ERP waves for ponel-PANEL and pinel- $P A N E L)$. Finally, we expect a different time course of orthographic and phonological priming effects, and this difference may reveal when phonological conversion is accomplished in normal reading. The idea is that N250 amplitude should be modulated by orthographic primes (see Grainger, Kiyonaga, et al., 2006). One question here is whether the tightly controlled phonological primes in the present experiment also show an N250 effect (with a different topology) or whether the onset of the phonological effect occurs later in processing.

\section{Method}

\section{Participants}

Thirty-two undergraduate students (21 women and 11 men) from the University of La Laguna participated in the experiment in exchange for course credit. All of them were native Spanish speakers, with no history of neurological or psychiatric impairment and with normal or corrected-to-normal vision. Ages ranged from 18 to 32 years $($ mean $=21.1$ years, $S D=3.6)$. All participants were right-handed, as assessed with an abridged Spanish version of the Edinburgh Handedness Inventory (Oldfield, 1971).

\section{Materials}

The experimental targets were 90 Spanish words of five or six letters. The mean number of letters was 5.7. All began with the consonant letter $c$ (mean occurrence per million: 36; range 1-417 in the Spanish database, Sebastián-Gallés, Martí, Carreiras, \& Cuetos, 2000). The mean number of substitution neighbors was less than 1.0 in the B-Pal database (Davis \& Perea, 2005). The targets were presented in uppercase and were preceded by nonword primes in lowercase that defined three conditions: (a) the prime was the same as the target except for a change of the second letter (always a vowel), but only the vowel phoneme changed (e.g., conal-CANAL); (b) the prime was the same as the target except for a change of the second letter (always a vowel) so that the prime and target differed only in the second letter, but the initial consonant phoneme changed as well as the vowel phoneme (e.g., cinal-CANAL); and (c) the prime was the same as the target except for a change of the initial two letters (a consonant and vowel) but the initial consonant phoneme did not change, so that only the vowel phoneme changed (e.g., konal-CANAL). We will term the first condition the phoneme and grapheme same condition, the second condition the phoneme change condition, and the third condition the grapheme change condition. These labels apply to the initial letter and phoneme; in all three conditions, the vowel phoneme and grapheme changed. The mean log bigram frequencies for the two initial letters in the phoneme and grapheme same and the phoneme change conditions were 2.8 versus 2.7 (token count), respectively; the initial bigram frequencies for the grapheme change condition are very low (0.1) because the letter $k$ is rather uncommon in Spanish.

For the key controls, we selected a set of 60 words of five and six letters (the mean number of letters was 5.5) that began with a consonant letter other than $C$ or $G$, so that the change of vowel did not alter the pronunciation of the prior consonant (mean frequency: 34 per million). The mean number of word neighbors was also less than one. The first two prime-target conditions for the control targets were orthographically the same as with the experimental targets (e.g., pinel-PANEL, ponel-PANEL); note that in both conditions, the primes shared all but one phoneme and all but one letter with the target (e.g., pinel and ponel each share five letters and five phonemes with $P A N E L$ ). The mean log bigram frequencies for the two initial letters in these two priming conditions were 2.6 versus 2.5 (token count), respectively. In addition, we selected 30 words preceded by an unrelated nonword target (e.g., sulor-PANEL). Note that this latter condition was put in to keep counterbalancing simple but we are going to ignore it henceforth.

Finally, 180 nonwords of five and six letters were created for the purposes of the lexical decision task. Half of the nonwords had the consonant $c$ as the first letter (experimental targets) and the other half has a different consonant letter as the first letter (control targets). The priming conditions for the nonword targets were analogous to those of the word targets (e.g., cubur-COBUR, cibur-COBUR, kobur-COBUR for the experimental targets; futul-FOTUL, fitul-FOTUL, lafer-FOTUL, for the control targets). A counterbalanced design was used in which three sets of materials were constructed so that each (word or nonword) target appeared once in each, but each time in a different priming condition. Different groups of participants were used for each of the three sets of materials.

\section{Procedure}

Participants sat comfortably in a darkened sound-attenuated chamber. All stimuli were presented on a high-resolution monitor that was positioned at eye level $80-90 \mathrm{~cm}$ in front of the participant. All targets were displayed in white uppercase Arial 24 point type against a dark-gray background. Primes were displayed in lowercase. Participants performed a lexical decision task: They were instructed to press one of two buttons on the response pad to indicate whether the letter string was a legitimate Spanish word or not. A response button was positioned beneath each thumb. For half of the participants the right button was used to signal the "yes" response and left button was assigned the "no" response. For the remaining participants the assignment was reversed. Each trial began with a row of hash marks ("\#\#\#\#\#\#"), which appeared in the center of the screen and remained there for $500 \mathrm{~ms}$. A prime word was then displayed for $47 \mathrm{~ms}$, followed by the target item, which was displayed for 400 $\mathrm{ms}$. The trial ended either with the participant's response or at $3000 \mathrm{~ms}$ after the presentation of the word if the participant failed to respond. The intertrial interval varied randomly between 1300 and $2300 \mathrm{~ms}$. Participants reported no awareness of the lowercase stimuli when asked after the experiment. All items were presented in a different random order for each participant in five different blocks, with a break of a few minutes between blocks in which the participant could rest and the impedances were checked.

Twenty-four warm-up trials, containing different stimuli from those used in the experimental trials, were provided at the beginning of the session. Participants were asked to avoid eye 
movements and blinks during the interval when the row of hash marks was not present and they were directed to favor accuracy over speed in their responses. Each session lasted approximately $1 \mathrm{~h} 15 \mathrm{~min}$.

\section{EEG Recording and Analyses}

Scalp voltages were collected from $58 \mathrm{Ag} / \mathrm{AgCl}$ electrodes that were mounted in an elastic cap (ElectroCap International, Eaton, $\mathrm{OH} ; 10-10$ system). Figure 1 shows the schematic distribution of the recording sites. Linked earlobes were used as reference. Eye movements and blinks were monitored with six additional electrodes providing bipolar recordings of the horizontal and vertical electrooculogram (EOG). Interelectrode impedances were kept below $10 \mathrm{k} \Omega$. EEG was filtered with an analog bandpass filter of $0.01-100 \mathrm{~Hz}$ and a digital $15=\mathrm{Hz}$ lowpass filter was applied before analysis. The signals were sampled continuously throughout the experiment with a sampling rate of $250 \mathrm{~Hz}$.

As in prior research with the masked priming technique, the focus was on the word targets. Only trials on which the response was correct and which were free of ocular artifacts (blinks and eye movements) and muscular artifacts were averaged and analyzed (more than $89 \%$ of word trials). Epochs of the EEG up to $800 \mathrm{~ms}$ after the onset of the target word were the primary data. The baseline correction was performed using the average EEG activity in the $250 \mathrm{~ms}$ preceding the onset of the target as a reference signal value; we also examined ERPs using baselines calculated during the $200 \mathrm{~ms}$ immediately preceding prime and the outcome was the same as the one presented here. Separate ERPs were formed for each of the experimental conditions, each of the subjects, and each of the electrode sites. Six regions of interest, three in each hemisphere, were constructed, and the response reported in each region is the mean response of the set of electrodes. The regions were (see electrode numbers in Figure 1) left anterior (F1, F3, F5, C1A, C3A, C5A), left central (C1, C3, C5, C1P, C3P, TCP1), left posterior (P1, P3, P5, P1P, P3P, $\mathrm{CB} 1$ ), right anterior (F2, F4, F6, C2A, C4A, C6A), right central (C2, C4, C6, C2P, C4P, TCP2), and right posterior (P2, P4, P6, $\mathrm{P} 2 \mathrm{P}, \mathrm{P} 4 \mathrm{P}, \mathrm{CB} 2)$.

Mean amplitudes were obtained for different time windows. For each window, two repeated-measures analyses of variance (ANOVAs) were performed. The first ANOVA was performed including electrode regions (anterior, central, and posterior), hemisphere (left/right), prime-target relatedness (phonemechange vs. phoneme- and grapheme-same) and type of target (experimental vs. control) as factors to test for phonological effects while controlling for orthography. A second ANOVA was performed including electrode regions (anterior, central, and posterior), hemisphere (left/right), and prime-target relatedness (grapheme-change vs. phoneme- and grapheme-same) as factors to test for orthographic effects while controlling for phonology. When appropriate, critical values were adjusted using the Greenhouse and Geisser (1959) correction for violation of the assumption of sphericity. Effects for the electrode region factor or for the hemisphere factor are only reported when they interact with the experimental manipulations.

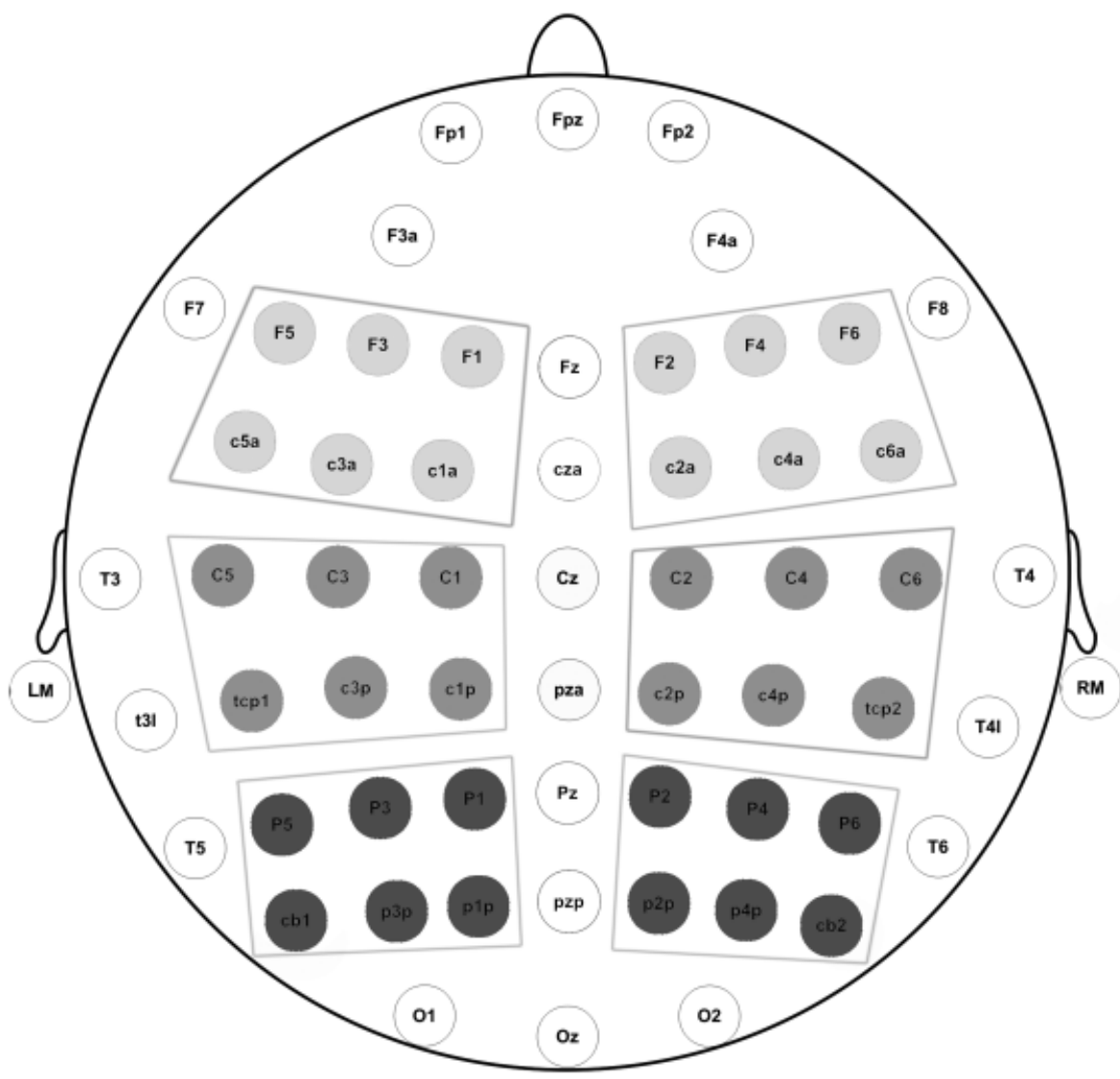

Figure 1. Schematic flat representation of the 58 electrode positions from which EEG activity was recorded (the front of the head is at the top). The electrodes grouped are those analyzed in the six critical regions. 


\section{Results}

\section{Behavioral Measures}

Incorrect responses (5.4\% of the data for word targets) and reaction times less than $250 \mathrm{~ms}$ or greater than $1500 \mathrm{~ms}$ (fewer than $2.5 \%$ of the responses to word targets) were excluded from the latency analysis. The mean latencies for correct responses and error rates are presented in Table 1. (The data from nonwords will not be considered further.) To examine the effect of phonology, ANOVAs based on the participants' response latencies and percentage error were conducted based on a 2 (Prime-Target Relatedness: phoneme-change, phoneme-same) $\times 2$ (Type of Target: experimental, control) within-subject design. To examine the effect of orthography, we compared the phoneme and grapheme-same conditions versus the grapheme-change condition for the experimental targets.

Effect of phonology. The ANOVA on the lexical decision times showed a main effect of type of target (with faster response times for the control targets), $F(1,31)=7.72, p<.01$. The main effect of prime-target relatedness was not significant, $F<1$. The data of greatest theoretical importance were an interaction reflecting a phonological priming effect: For the experimental targets responses were $9 \mathrm{~ms}$ faster when preceded by the phoneme-same primes than when preceded by the phoneme-change primes (e.g., conal-CANAL faster than cinal-CANAL), $F(1,31)=$ $3.16, p=.08$, whereas the analogous effect for the control targets was $-1 \mathrm{~ms}$. (Note that even though the $F$ ratio for the PrimeTarget Relatedness $\times$ Type of Target interaction was not significant, $F[1,31]=1.80, p=.19$, the obtained pattern perfectly mimics the experiment conducted by Pollatsek et al., 2005, which also used a 50-ms stimulus onset asynchrony [SOA].) The ANOVA on the error data only showed an overall effect of type of target (with fewer errors for the control targets), $F(1,31)=7.19, p<.015$.

Effect of orthography. As can be seen in Table 1, there was virtually no orthographic priming effect on either the response time $(-1 \mathrm{~ms})$ or the error rate $(+0.1 \%)$, all $F \mathrm{~s}<1$.

\section{Electrophysiological Measures}

Only ERP waves for correct target words were analyzed because none of the effects for pseudowords either in the present experiment or in previous experiments using these same stimuli (Pollatsek et al., 2005) were significant. Indeed, masked priming effects on pseudoword targets in the lexical decision task tend to be small and unreliable (Forster, 1998; Norris \& Kinoshita, 2008).

Only comparisons relevant to the phonological and orthographic effects will be presented here, as they are the central focus of this article. ERP grand averages time-locked to the onset of the target words are represented in Figures 2 and 3 over the six regions of interest. Figure 2 shows the phonological contrasts, whereas Figure 3 shows the orthographic contrasts. Visual inspection of Figure 2 reveals differences in amplitude for the experimental conditions (phoneme same vs. different) but not for the two controls. In particular, the amplitude of the phoneme different condition shows a larger amplitude starting around 350 ms. Visual inspection of Figure 3 shows differences between the grapheme different and the grapheme same conditions starting as early as $150 \mathrm{~ms}$. To investigate whether these differences were statistically significant, we defined three different windows of analyses (150-250, 250-350, and 350-550 ms) based on visual inspection and the previous work by Grainger, Kiyonaga, et al. (2006). (The same windows as in that study were also used so that the two sets of results can be easily compared.) See Figure 4 or a topographical distribution of the effects in these three windows.

Phonological effects. The ANOVA on the average values of the 150-250- and 250-350-ms time epochs did not reveal any significant difference. Importantly, the ANOVA on the average values of the 350-550-ms time window showed an interaction between prime-target relatedness (phoneme-change vs. phoneme and grapheme-same) and type of target (experimental vs. control) $F(1,31)=6.10, p<.02$. This interaction reflected, for the experimental targets, a significantly larger negative amplitude in the phoneme-change than in the phoneme-same condition $F(1,31)=5.23, p<.05$. In contrast, there were no signs of a similar effect between the two analogous conditions for the control targets $(F<1)$. No other effects were significant.

Orthographic effects. The ANOVA on the average values of the 150-250-ms time epoch showed a larger negative amplitude for the grapheme-change than the grapheme-same condition $F(1,31)=3.97, p=.05$, and an interaction of this factor with hemisphere $F(1,31)=4.53, p<.05$ : The larger negative amplitude for the grapheme-change occurred to a larger degree in the left hemisphere $F(1,31)=5.64, p<.05$, than in the right hemisphere, $F(1,31)=2.40, p>.13$. In later time windows $(250-350$ and $350-550 \mathrm{~ms}$ ), the differences between the grapheme-change and the grapheme-same conditions were still present (see Figure 3 ), but the statistical analyses only reflected a marginally significant difference, $F(1,31)=3.26, p=.08$ and $F(1,31)=3.12$, $p=.08$, respectively.

\section{Discussion}

The present experiment provides clear evidence of phonological involvement with a brief SOA (and heavily masked primes) on the ERP waves. Importantly, unlike most experiments providing evidence for masked phonological priming, the critical compar-

Table 1. Mean Lexical Decision Times (in Milliseconds) and Percentage of Errors (in Parentheses) on Words and Pseudowords

\begin{tabular}{|c|c|c|c|c|c|}
\hline & \multicolumn{3}{|c|}{ Type of prime } & \multicolumn{2}{|c|}{ Priming effect } \\
\hline & Phoneme- and grapheme-same & Phoneme-change & Grapheme-change & Phonology & Orthography \\
\hline \multicolumn{6}{|l|}{ Word trials } \\
\hline Experimental & $693(6.2)$ & $702(6.3)$ & $692(6.3)$ & $9(0.1)$ & $-1(0.1)$ \\
\hline Control & $685(4.4)$ & $684(4.6)$ & $703(4.7)^{\mathrm{a}}$ & $-1(0.2)$ & \\
\hline \multicolumn{6}{|l|}{ Nonword trials } \\
\hline Experimental & $765(2.9)$ & $768(2.2)$ & $773(3.6)$ & $3(-0.7)$ & $8(0.7)$ \\
\hline Control & $761(4.1)$ & $765(3.1)$ & $765(4.0)^{\mathrm{a}}$ & $4(-1.0)$ & \\
\hline
\end{tabular}

"The "grapheme-change" condition for the control targets is an unrelated prime condition. 

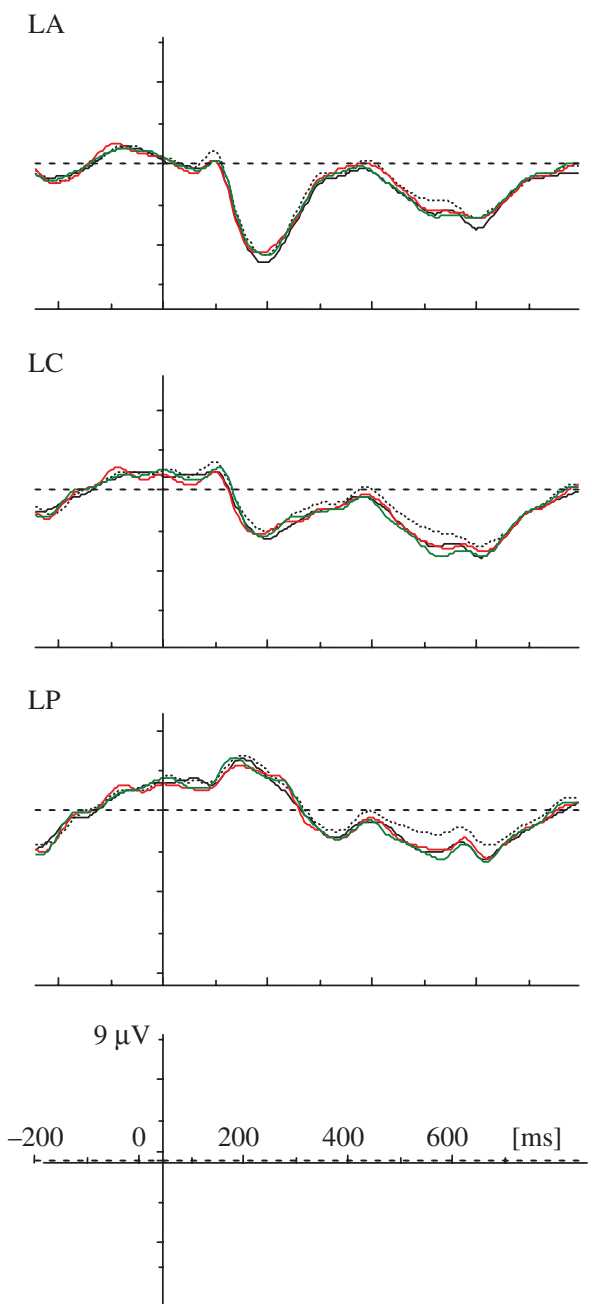

RA

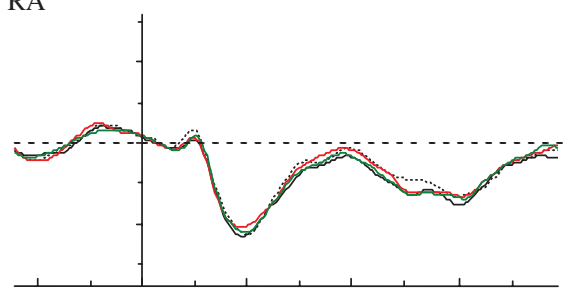

$\mathrm{RC}$

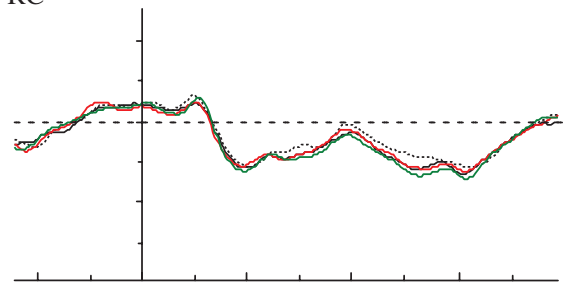

RP

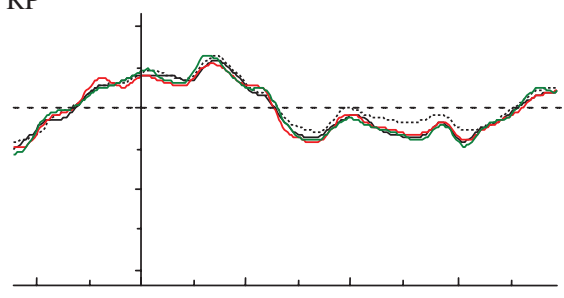

conal - CANAL cinal - CANAL ponel - PANEL pinel-PANEL

Figure 2. ERP waves corresponding to the phonological contrasts (phoneme same vs. different) of the averaged amplitude values for the six regions of interest, where six electrodes are pooled for each measurement: left anterior (F5, F3, F1, C5A, C3A, C1A), left central (C5, C3, C1, TCP1, C3P, C1P), left posterior ( $\mathrm{P} 5$, P3, P1, CB1, P3P, P1P), right anterior (F2, F4, F6, C2A, C4A, C6A), RIGHT CENTRAL (C2, C4, C6, C2P, C4P, TCP2), and right posterior (P2, P4, P6, P2P, P4P, CB2). Negative voltages are plotted up.

ison was between sets of primes that had the same orthographic similarity to the target words but differed in phonological similarity (e.g., conal-CANAL vs. cinal-CANAL vis á vis ponel$P A N E L$ vs. pinel-PANEL). Masked phonological priming effects started to occur in the 350-550-ms time window. Note that the difference in the phonological relations of primes to the target began later than the difference in the orthographic relations of the prime to the target (e.g., conal-CANAL vs. konal$C A N A L)$. Although the orthographic priming effect was mainly observed in the 150-250-ms epoch, there were hints that it continued into the later epoch. Finally, the present experiment is a also a clear demonstation that ERP waves can reveal effects that may not be reliable in behavioral measures - note that response times give the researcher only one data point at the end of processing and this may be less sensitive to the manipulation than the examination of the time course of the ERP waves (e.g., see Carreiras, Vergara, \& Perea, 2009, for another dissociation of ERP and RTs in a masked priming paradigm). However, although the phonological effect in the response times in the present experiment was marginally significant, it was approximately the same size as the significant effect observed by Pollatsek et al. (2005).
The time courses of the orthographic and phonological effects we observed were similar (to some degree) to those found previously by Grainger, Kiyonaga, et al. (2006) in a recent ERP study (see Figure 4). Both studies thus suggest that there is an early phase of orthographic processing that is not influenced by phonology that is followed by rapid activation of phonological codes. However, there are two important caveats to keep in mind. First, as indicated earlier, orthographic differences in all studies are confounded with differences in relatively low-level visual properties of the letters (e.g., $k$ is visually more different from $C$ than $c$ is); furthermore, in most previous masked orthographic priming experiments, the typical comparison has been between an orthographically related prime and an unrelated prime. Second, our behavioral data indicate that there were no signs of an "orthographic" priming effect in the response times. This raises the question of whether there is an early visual or orthographic effect that is more or less irrelevant to word identification. That is, there may be some early and low-level system that notices the visual or orthographic difference (or the size of the difference) between prime and target that may be relevant to the attentional system, but it may not be entirely central to word 
LA

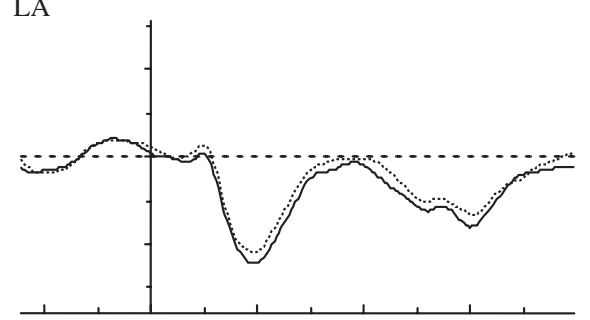

LC

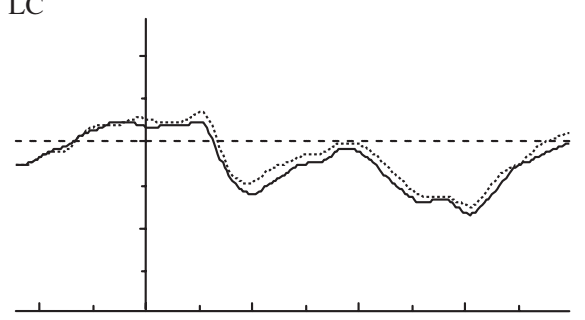

LP
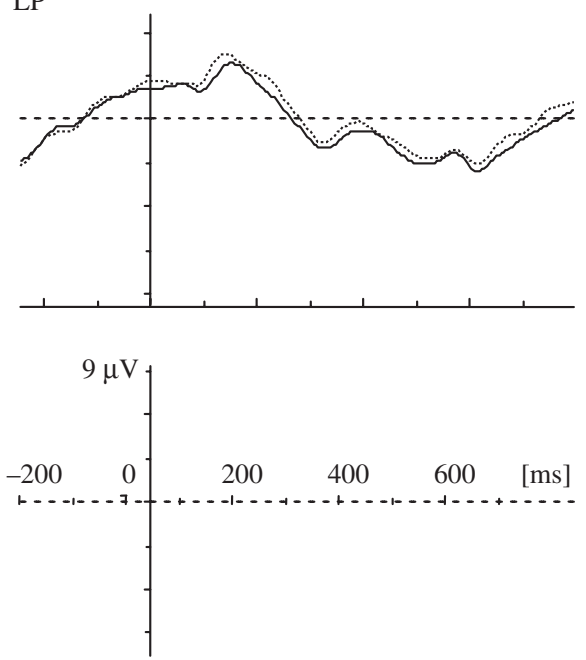

RA

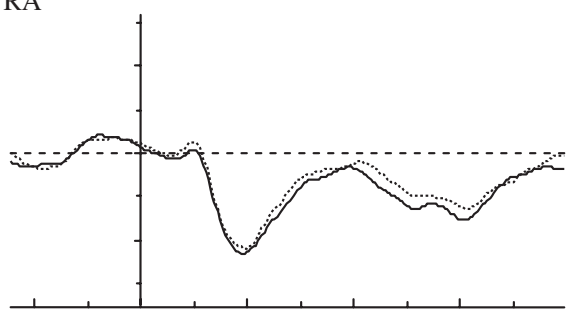

RC

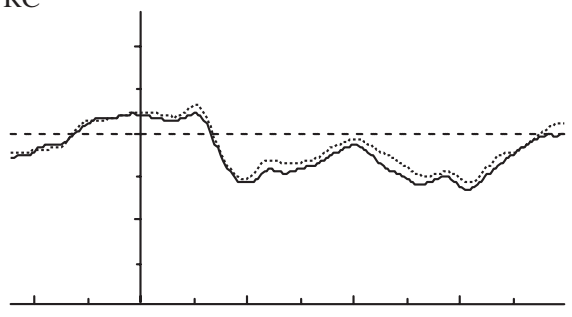

RP

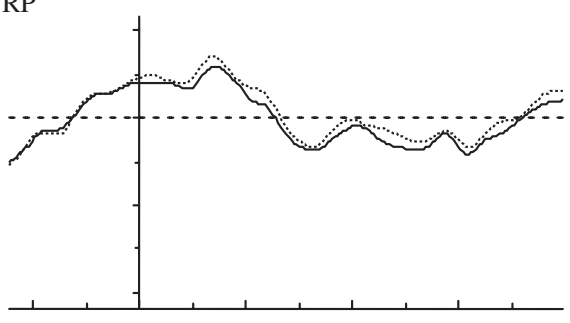

conal - CANAL

konal - CANAL

Figure 3. ERP waves corresponding to the orthographic contrasts (grapheme same vs. different) of the averaged amplitude values for the six regions of interest, where six electrodes are pooled for each measurement: left anterior (F5, F3, F1, C5A, C3A, C1A), left central (C5, C3, C1, TCP1, C3P, C1P), left posterior (P5, P3, P1, CB1, P3P, P1P), right anterior (F2, F4, F6, C2A, C4A, C6A), right central (C2, C4, C6, C2P, C4P, TCP2), and right posterior (P2, P4, P6, P2P, P4P, CB2). Negative voltages are plotted up.

identification. In fact, previous work (Chauncey, Holcomb, \& Grainger, 2008) has documented that changing the font was found to affect repetition priming in the N/P150 component (this component was defined for a window between 90 and $180 \mathrm{~ms}$ ), but not in later components such as the N250. In addition, this effect was also significant between 100 and $250 \mathrm{~ms}$ when analyses were performed defining windows of $50 \mathrm{~ms}$. In the present experiment, the orthographic effect was significant in a window of $150-250 \mathrm{~ms}$ and marginally significant in later windows. Thus, we may have been capturing the combined effect of orthographic and visual processes. Further research will be needed to disentangle these two effects.

As indicated earlier, activation of phonological codes has been commonly obtained with the masked priming paradigm and brief prime durations (e.g., Álvarez, Carreiras, \& Perea, 2004; Carreiras, Ferrand, Grainger, \& Perea, 2005; Frost, Ahissar, Gotesman, \& Tayeb, 2003; Lukatela \& Turvey, 1994; Perfetti \& Bell, 1991; Pollatsek et al., 2005; Rastle \& Brysbaert, 2006). Therefore, our finding of a masked phonological priming effect is not an isolated finding; indeed, it appears in a variety of languages. Nonetheless, our results clearly differ from other studies with respect to "orthographic" priming effects in the behavioral data in that such effects have been consistently obtained in many studies in contrast to the present study. However, there is a key difference between the present study and the prior ones: In the present study, the orthographic difference was not confounded with a phonological difference (e.g., kanal and canal are homophones). The inference of an earlier orthographic effect in the behavioral data (when comparing a related pair like lonc-LONG vs. an unrelated pair like tabe-LONG; e.g., Ferrand \& Grainger, $1992,1994)$ is usually drawn from the finding that orthographic effects are observed with shorter prime durations than are phonological effects. As argued above, in the other studies, orthographic and phonological differences were confounded in the "orthographic" manipulation; thus, the robust "orthographic" effect at prime durations that were too short in which to observe phonological effects could have either been combined orthographic/phonological effects or, in some cases, merely effects of 
ORTHOGRAPHY
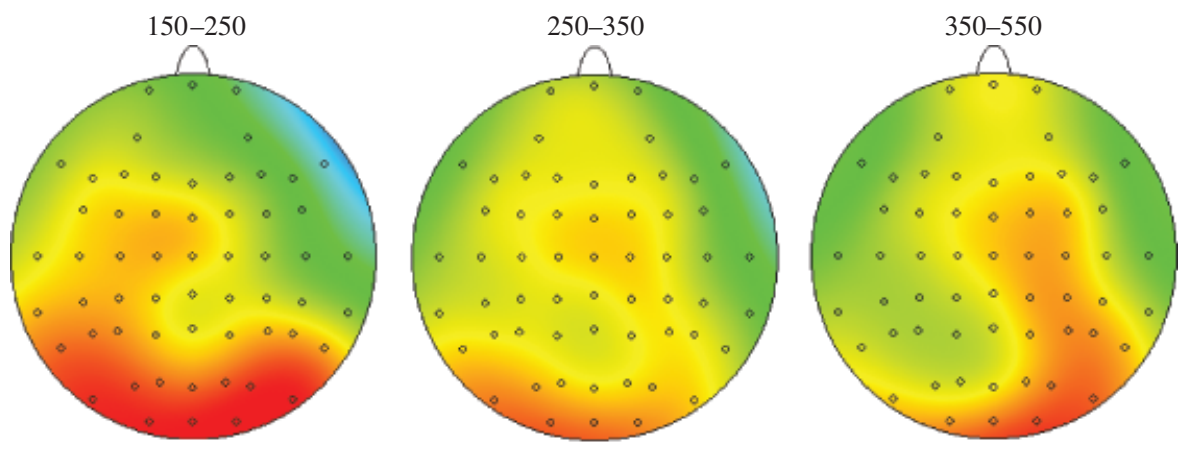

$\underline{\text { PHONOLOGY }}$
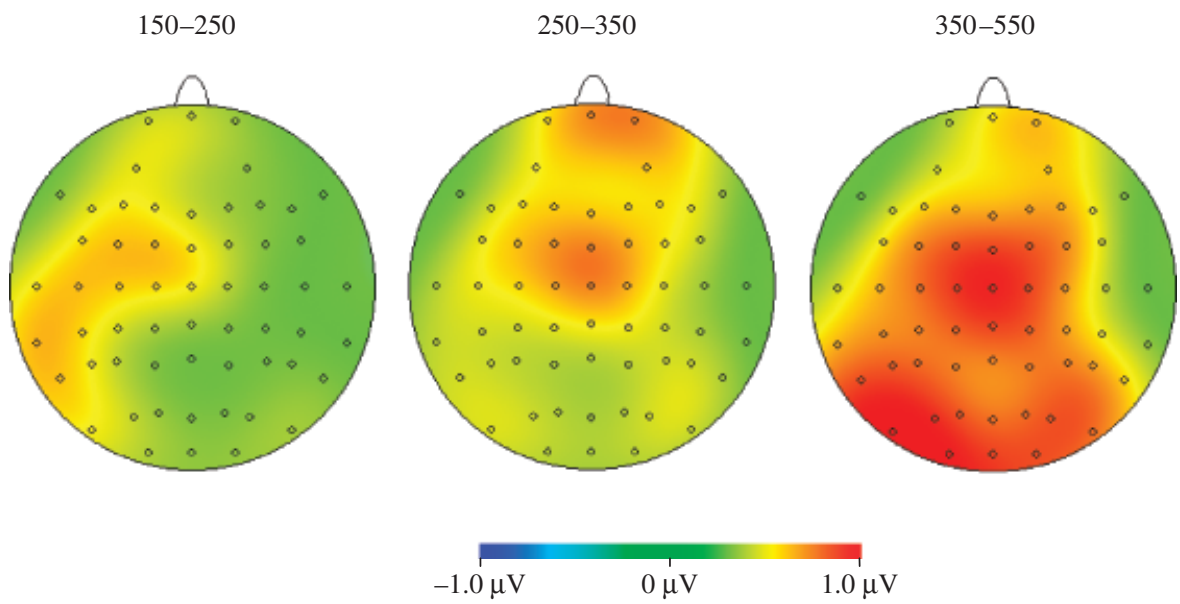

$0 \mu \mathrm{V}$

Figure 4. Topographical distribution of the orthographic and phonological priming effects in the three windows of analyses.

greater phonological changes than occurred in the phonological condition - or even more visual similarity in the orthographic than in the phonological pairs.

Our ERP results, however, do differ from those of Grainger, Kiyonaga, et al. (2006) in one respect. They found that their orthographic manipulation had an effect in the 150-250-ms window, as in the present experiment, but their phonological manipulation had an effect in the 250-350 ms window, whereas we only found an effect of phonology in the 350-550-ms time window. Thus, there was a longer delay (around $100 \mathrm{~ms}$ ) between orthographic and phonological ERP effects than observed by Grainger, Kiyonaga, et al. (2006), which was around $50 \mathrm{~ms}$. However, there were important differences in the procedure and the materials between the two studies that may be responsible for this difference in the results.

First, Grainger, Kiyonaga, et al. (2006) used a backward mask of seven random consonants (e.g., CFTRPQB) that immediately replaced the prime and lasted for $17 \mathrm{~ms}$, whereas in our experiment the target replaced immediately the prime. Thus, it could be argued that in the Grainger, Kiyonaga, et al. (2006) study there was more time available for processing the prime (even though backwardly masked). Second, they used a semantic categorization task, whereas we used a lexical decision task. It may well be the case that differences in time course for the orthographic and phonological codes can be caused by the specific processing requirements of the two tasks. Third, as argued above, the orthographic and phonological manipulations were different. For the phonological manipulation, Grainger, Kiyonaga, et al. (2006) compared words like $B R A I N$ preceded by (a) pseudohomophones (brane) or (b) control words (brant). In our paradigm, we compared words like $C A N A L$ preceded by (a) a condition of phoneme-change (cinal-CANAL) or (b) a condition with the same phoneme and same grapheme (conal-CANAL). Indeed, the effects of the phonological manipulation in the Grainger, Kiyonaga, et al. (2006) study may not be solely due to difference in terms of phonology (between brane and brant as primes for $B R A I N$ ). There are also two phonological differences between the phonological manipulations in the two experiments. First, our phonological difference could be considered more subtle: In the Grainger, Kiyonaga, et al. (2006) experiment, the related prime brane and the target $B R A I N$ share exactly the same phonology, whereas none of our primes (e.g., conal-CANAL vs. cinal-CANAL) were homophones of the target, and thus their phonological manipulation may have been stronger than ours. Second, because the phonology from brane is an exact match of $B R A I N$, the word form may have been preactivated and its processing/recognition would elicit earlier and smaller negativities than BRAIN preceded by brant. In contrast, although conal is more phonologically similar to $C A N A L$ than cinal is, it does not exactly match $C A N A L$. Thus, activation of the word unit corresponding to $C A N A L$ may not only be lower, but may also compete with other word forms activated by the prime conal, 
which may be time-consuming and produce a later shift. Third, because in the Grainger, Kiyonaga, et al. (2006) study the primes were pseudohomophones, these items work phonologically as words, and therefore there should be semantic activation from these primes. However, semantic activation does not seem to be an issue in the present study.

The present data also have implications for recently proposed input coding schemes in visual-word recognition (e.g., the SOLAR model, Davis, 1999; the SERIOL model, Whitney, 2001; overlap model, Gomez, Ratcliff, \& Perea, 2008; and the openbigram model, Grainger \& van Heuven, 2003). Unlike the "channel-specific" input coding scheme used by the interactive activation model and its successors (e.g., Coltheart, Rastle, Conrad, Langdon, \& Ziegler, 2001), the new input coding schemes can successfully cope with relative-position coding effects (e.g., blcny activates BALCONY; see Grainger, Granier, Farioli, Van Assche, \& van Heuven, 2006) and with transposedletter effects (e.g., caniso activates CASINO; e.g., Perea \& Lupker, 2004). One limitation of these new input coding schemes, however, is that, in their current version, they focus on orthography rather than phonology. Indeed, only the SERIOL model includes a phonological module. The SERIOL model assumes the presence of a phonological route that operates with biphones in the same way that the orthographic route operates with bigrams (Whitney \& Cornelissen, 2005), and conal and $C A N A L$ share a higher number of biphones than cinal and $C A N A L$. Although specific simulations on an implemented ver- sion of the model are necessary, it seems that the SERIOL model can, in principle, accommodate the present findings (i.e., a faster activation in the orthographic pathway that is followed by the activation in the phonological pathway). This can be done by assuming that the phonological pathway processes the information in a sequential way (see Whitney \& Cornelissen, 2008). Clearly, the other input coding schemes (e.g., the SOLAR model; Davis, 1999; the overlap model, Gomez et al., 2008; and the open-bigram model, Grainger \& van Heuven, 2003) need to be expanded to deal with phonological processing (see Perea \& Carreiras, 2008).

In sum, the present findings are consistent with the view that phonology plays an important role during reading. It is important to stress that such phonological activation can occur when using an extreme orthographic overlap via tight controls (e.g., conal-CANAL vs. cinal-CANAL vis á vis ponel-PANEL vs. pinel$P A N E L$ ). (Of course, one limitation of our procedure is that it is restricted to targets containing context-sensitive letters, such as the letter $c$.) Furthermore, our findings are consistent with previous homophone priming experiments that show early involvement of phonological processing in languages as different as English (e.g., Grainger, Kiyonaga, et al., 2006; Pollatsek et al., 1992), Hebrew (e.g., Frost et al., 2003), or French (Ferrand \& Grainger, 1992, 1994). Finally, the present finding have clear implications for the coding scheme of the computational models of visual-word recognition, which need to account for a sequential activation of orthographic and phonological codes during word reading.

\section{REFERENCES}

Álvarez, C., Carreiras, M., \& Perea, M. (2004). Are syllables phonological units in visual word recognition? Language and Cognitive Processes, 19, 427-452.

Barber, H., \& Kutas, M. (2007). Interplay between computational models and cognitive electrophysiology in visual word recognition. Brain Research Reviews, 53, 98-123.

Carreiras, M., Ferrand, L., Grainger, J., \& Perea, M. (2005). Sequential effects of masked phonological priming. Psychological Science, 16, 585-589.

Carreiras, M., Gillon-Dowens, M., Vergara, M., \& Perea, M. (2009). Are vowels and consonants processed differently? ERP evidence with a delayed letter paradigm. Journal of Cognitive Neuroscience, 21, 275288.

Carreiras, M., Vergara, M., \& Barber, H. (2005). Early ERP effects of syllabic processing during visual word recognition. Journal of Cognitive Neuroscience, 17, 1803-1817.

Carreiras, M., Vergara, M., \& Perea, M. (2009). ERP correlates of transposed-letter priming effects: The role of vowels versus consonants. Psychophysiology, 46, 34-42.

Chauncey, K., Holcomb, P. J., \& Grainger, J. (2008). Effects of stimulus font and size on masked repetition priming: An ERP investigation. Language and Cognitive Processes, 23, 183-200.

Coltheart, M., Rastle, K., Conrad, P., Langdon, R., \& Ziegler, J. (2001). DRC: A dual route cascaded model of visual word recognition and reading aloud. Psychological Review, 108, 204-256.

Davis, C. J. (1999). The Self-Organising Lexical Acquisition and Recognition (SOLAR) model of visual word recognition. Unpublished doctoral dissertation. University of South Wales. Retrieved from http:// www.pc.rhul.ac.uk/staff/c.davis/Thesis.

Davis, C. J., \& Perea, M. (2005). BuscaPalabras: A program for deriving orthographic and phonological neighborhood statistics and other psycholinguistic indices in Spanish. Behavior Research Methods, 37, 665-671.

Ferrand, L., \& Grainger, J. (1992). Phonology and orthography in visual word recognition: Evidence from masked nonword priming. Quarterly Journal of Experimental Psychology, 45A, 353372 .
Ferrand, L., \& Grainger, J. (1994). Effects of orthography are independent of phonology in masked form priming. Quarterly Journal of Experimental Psychology, 47A, 365-382.

Forster, K. I. (1998). The pros and cons of masked priming. Journal of Psycholinguistic Research, 27, 203-233.

Frankish, C., \& Turner, E. (2007). SIHGT and SUNOD: The role of orthography and phonology in the perception of transposed letter anagrams. Journal of Memory and Language, 56, 189-211.

Frost, R., Ahissar, M., Gotesman, R., \& Tayeb, S. (2003). Are phonological effects fragile? The effect of luminance and exposure duration on form priming and phonological priming. Journal of Memory and Language, 48, 346-378.

Gomez, P., Ratcliff, R., \& Perea, M. (2008). A model of letter position coding: The overlap model. Psychological Review, 115, 577-601.

Grainger, J., \& Ferrand, L. (1994). Phonology and orthography in visual word recognition: Effects of masked homophone primes. Journal of Memory and Language, 33, 218-233.

Grainger, J., Granier, J. P., Farioli, F., Van Assche, E., \& van Heuven, W. (2006). Letter position information and printed word perception: The relative-position priming constraint. Journal of Experimental Psychology: Human Perception and Performance, 32, 865-884.

Grainger, J., Kiyonaga, K., \& Holcomb, P. J. (2006). The time course of orthographic and phonological code activation. Psychological Science, 17, 1021-1026.

Grainger, J., \& van Heuven, W. J. B. (2003). Modeling letter position coding in printed word perception. In P. Bonin (Ed.), The mental lexicon (pp. 1-23). New York: Nova Science.

Greenhouse, S., \& Geisser, S. (1959). On methods in the analysis of profile data. Psychometrika, 24, 95-112.

Holcomb, P. J., \& Grainger, J. (2006). On the time course of visual word recognition: An event-related potential investigation using masked repetition priming. Journal of Cognitive Neuroscience, 18, 1631-1643.

Holcomb, P. J., \& Grainger, J. (2007). Exploring the temporal dynamics of visual word recognition in the masked repetition priming paradigm using event-related potentials. Brain Research, 1180, 39-58.

Lee, H., Rayner, K., \& Pollatsek, A. (1999). The time course of phonological, semantic, and orthographic coding in reading: Evidence 
from the fast priming technique. Psychonomic Bulletin \& Review, 6 , 624-634.

Lukatela, G., \& Turvey, M. T. (1994). Visual access is initially phonological: 2. Evidence from phonological priming by homophones, and pseudohomophones. Journal of Experimental Psychology: General, 123, 331-353.

Norris, D., \& Kinoshita, S. (2008). Perception as evidence accumulation and Bayesian inference: Insights from masked priming. Journal of Experimental Psychology: General, 137, 434-455.

Oldfield, R. C. (1971). The assessment and analysis of handedness: The Edinburgh inventory. Neuropsychologia, 9, 97-113.

Perea, M., \& Carreiras, M. (2006). Do transposed-letter similarity effects occur at a prelexical phonological level? Quarterly Journal of Experimental Psychology, 59, 1600-1613.

Perea, M., \& Carreiras, M. (2008). Do orthotactics and phonology constrain the transposed-letter effect? Language and Cognitive Processes, 23, 69-92.

Perea, M., \& Lupker, S. J. (2004). Can CANISO activate CASINO? Transposed-letter similarity effects with nonadjacent letter positions. Journal of Memory and Language, 51, 231-246.

Perfetti, C. A., \& Bell, L. C. (1991). Phonemic activation during the first $40 \mathrm{~ms}$ of word identification: Evidence from backward masking and priming. Journal of Memory and Language, 30, 473485.

Pollatsek, A., Lesch, M., Morris, R. K., \& Rayner, K. (1992). Phonological codes are used in integrating information across saccades in word identification and reading. Journal of Experimental Psychology: Human Perception and Performance, 18, 148-162.

Pollatsek, A., Perea, M., \& Carreiras, M. (2005). Does conal prime CANAL more than cinal? Masked phonological priming effects in Spanish with the lexical decision task. Memory and Cognition, 33, 557-565.

Rastle, K., \& Brysbaert, M. (2006). Masked phonological priming effects in English: Are they real? Do they matter? Cognitive Psychology, 53, $1-49$.

Sebastián-Gallés, N., Martí, M. A., Carreiras, M., \& Cuetos, F. (2000). LEXESP: Una base de datos informatizada del español [LEXESP: A computerized database of Spanish]. Barcelona, Spain: Edicions de la Universitat de Barcelona.

Whitney, C. (2001). How the brain encodes the order of letters in a printed word: The SERIOL model and selective literature review. Psychonomic Bulletin and Review, 8, 221-243.

Whitney, C., \& Cornelissen, P. (2005). Letter-position encoding and dyslexia. Journal of Research in Reading, 28, 274-301.

Whitney, C., \& Cornelissen, P. (2008). SERIOL reading. Language and Cognitive Processes, 23, 143-164.

Ziegler, J. C., Ferrand, L., Jacobs, A. M., Rey, A., \& Grainger, J. (2000). Visual and phonological codes in letter and word recognition: Evidence from incremental priming. Quarterly Journal of Experimental Psychology: Human Experimental Psychology, 53A, 671-692.

(Received June 11, 2008; ACCEPTED November 14, 2008) 